\begin{tabular}{ll}
\hline \hline MINING AND METALLURGY INSTITUTE BOR & ISSN: 2334-8836 \\
& UDK: 622 \\
\hline \hline
\end{tabular}

\title{
ARSENIC CONTENT IN THE LEAD FLOTATION CONCENTRATE IN DEPENDENCE ON NaCN DOSAGE ${ }^{* *}$
}

\begin{abstract}
Flotation properties of arsenopyrite are similar to the flotation properties of pyrite. Specifically, in the alkaline conditions ( $\mathrm{pH}$ value of pulp 7-11) and in the presence of xanthates as collectors, arsenopyrite can easily pass into concentrate. Therefore, if the ore contains arsenopyrite as supporting component, its flotation characteristics can affect the certain difficulties in the flotation of other minerals $(\mathrm{Pb}, \mathrm{Zn}, \mathrm{Sb}, \mathrm{W}$, etc.), because arsenic is considered as harmful element in almost all concentrates of metallic minerals. To the aim of obtaining high quality flotation concentrate, it is often necessary to depress arsenopyrite, which can be successfully achieved by applying of cyanides.

This paper presents the results of laboratory tests, performed in order to determine the possibility of obtaining a lead concentrate with low content of arsenic. Ore sample, which was used in this flotation testing, contained a large amount of arsenopyrite (over $40 \%$ by mass). The results showed that arsenic content in the rough lead concentrate can be decreased up to ten times compared to its content in the ore, using NaCN as depressant.
\end{abstract}

Keywords: arsenic, sodium cyanide, flotation concentrate

\section{INTRODUCTION}

Arsenic is considered as a harmful element in selective concentrates of lead and zinc $[1,2]$. The llowable arsenic content in these concentrates is expressed in tenth parts of a percent [4].

One of the most important arsenic minerals is arsenopyrite (FeAsS) which is often major gangue mineral in base metallic sulfide ores. Therefore, in the processing of polymetallic ores of lead, zinc, copper, pyrite and gold, it is very important to perform successful depression of arsenopyrite during the separation of selective concentrates. Draškić [3] points out that depression of arsenopyrite should be made at the beginning of selective concentration, because its depression in collective concentrates is much harder to achieve.

According to literature data, depression of arsenopyrite can be performed in a strong alkaline medium [6,7] and in the presence of oxidants, such as hydrogen peroxide $\left(\mathrm{H}_{2} \mathrm{O}_{2}\right)$, potassium permanganate $\left(\mathrm{KMnO}_{4}\right)$, manganese dioxide $\left(\mathrm{MnO}_{2}\right)$ and hypochlorous acid ( $\mathrm{HClO})[3,5]$. In addition, some authors suggest that arsenopyrite can be effectively depressed by sodium cyanide $(\mathrm{NaCN})[3,9]$. Furthermore, a mixture of ammoniummagnesium salts has been found to be an

\footnotetext{
* Mining and Metallurgy Institute Bor, srdjana.magdalinovic@irmbor.co.rs

** This work is the result of the Projects: TR 33023: "Development of Technologies for the Flotation Processing of Copper and Precious Metal Ores with the Aim to Increase the Technological Results" and TR 34006: "Mechanochemical Treatment of Insufficient Quality Mineral Resources" funded by the Ministry of Education, Science and Technological Development of the Republic of Serbia.
} 
effective depressant. Strontium and barium ions have been reported to promote the depression of arsenopyrite and inhibit activation by copper ions [5].

Arsenopyrite floats well using xanthate as a collector. Oxidation of xanthate ions $\left(\mathrm{X}^{-}\right)$to dixanthogen $\left(\mathrm{X}_{2}\right)$ is attributed as the mechanism through which the collector adsorbs at the arsenopyrite/aqueous solution interface with the surface of arsenopyrite acting as a catalyst for the oxidation reaction yielding $\mathrm{OH}^{-}$ions as reaction products [5]. Copper ions (if they are present in flotation pulp) activate the flotation of arsenopyrite, lowering the amount of xanthate needed for flotation $[3,5,8]$.
The aim of the experimental testing, the results of which are presented in this paper, was to obtain rough lead concentrate with the low content of arsenic.

\section{EXPERIMENTAL PROCEDURE}

\section{Raw material}

Sample of polymetallic sulphide ore was used in the overall tests. In order to achieve proper fineness, the sample was previously crushed in a laboratory jaw crusher, and after that ground in a laboratory ball mill. Chemical composition of the sample is shown in Table 1.

Table 1. Chemical composition of the sample

\begin{tabular}{|c|c|c|c|c|c|c|c|c|}
\hline Component & $\mathrm{Pb}$ & $\mathrm{Zn}$ & $\mathrm{Cu}$ & $\mathrm{As}$ & $\mathrm{Sb}$ & $\mathrm{S}$ & $\mathrm{Fe}$ & $\mathrm{SiO}_{2}$ \\
\hline Mass content, $\%$ & 7.25 & 0.70 & 0.15 & 19.45 & 0.047 & 16.92 & 16.34 & 32.62 \\
\hline
\end{tabular}

Based on the results, shown in Table 1, it can be concluded that the examined sample contains a high amount of arsenic, even $19.45 \%$.

Qualitative mineralogical analysis of the sample was performed using the microscope with reflected light in the air (AXIOSKOP 40 device, with a unit for photomicrography). Microscopic tests showed that the sample contained the following major sulphide minerals: arsenopyrite (43.53\%), pyrite $(34.71 \%)$, galena $(5.88 \%)$ and sphalerite (2.95\%). The other sulphides (chalcopyrite,

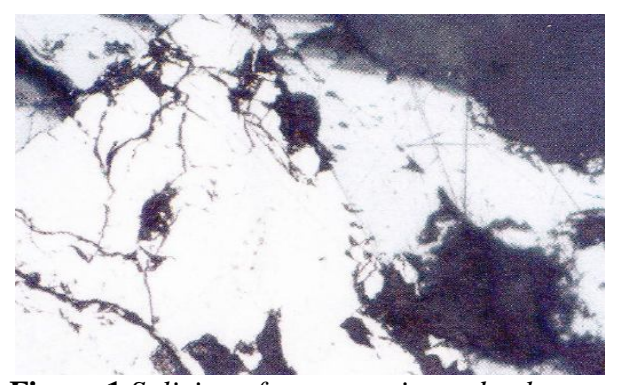

Figure 1 Splicing of arsenopyrite and galena $200 x$ enlargement, II $\mathrm{N}$ chalcocite) were presented in small quantities.

Arsenopyrite predominantly occurred in large crystal forms. In some arsenopyrite grains, a small embedded sphalerite grains could be observed. In addition, splicing of arsenopyrite with pyrite, galena and sphalerite were also noticed in the sample.

Galena occurs in the form of independent large grains. Small grains of galena are associated with pyrite and arsenopyrite.

Figures 1-6 illustrate the results of mineralogical analysis of the sample.

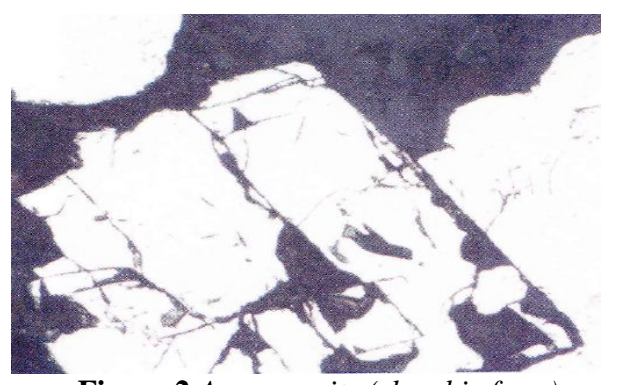

Figure 2 Arsenopyrite (rhombic form), 200x enlargement, II $N$ 


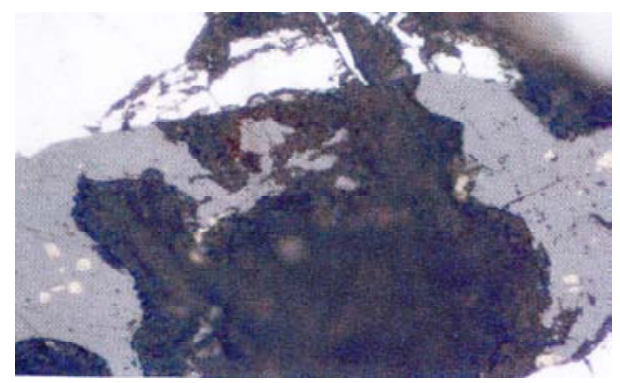

Figure 3 Separation of chalcopyrite in sphalerite, $400 x$ enlargement, II $\mathrm{N}$

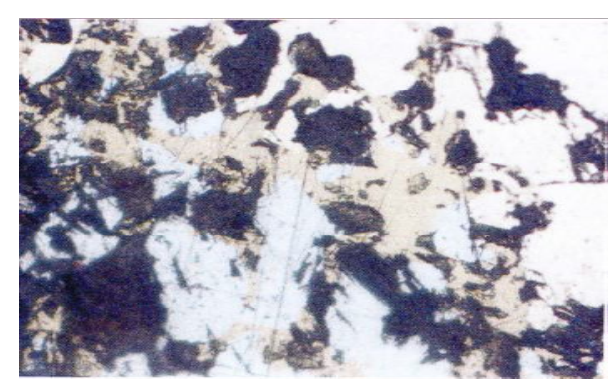

Figure 5 Splicing of pyrite, chalcopyrite and galena, 200x enlargement, II $\mathrm{N}$

For the purposes of flotation concentration tests, it was necessary to grind raw material to thesuitable fineness $(75 \%$ -

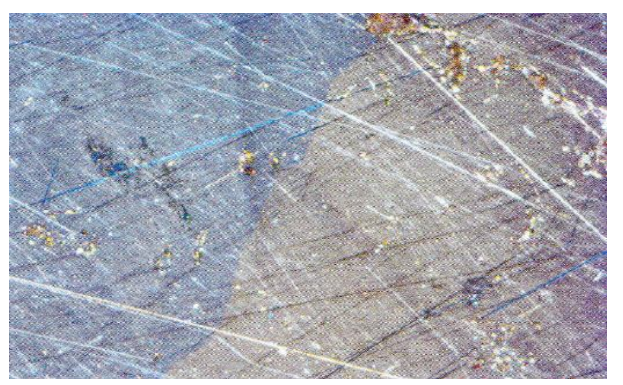

Figure 4 Arsenopyrite, 100x enlargement, $+N$

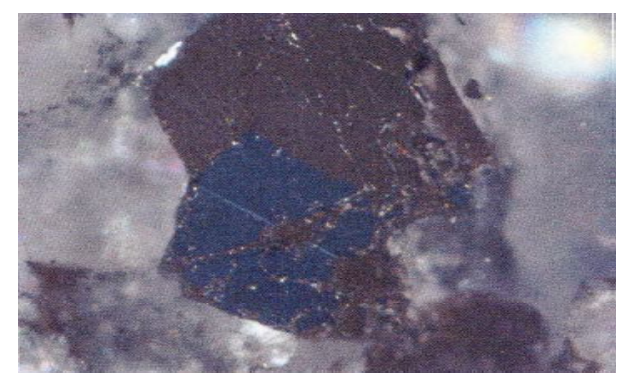

Figure 6 Arsenopyrite, 200x enlargement, $+N$

$0.075 \mathrm{~mm}$ ). Grain-size distribution of grinded sample is shown in Figure 7.

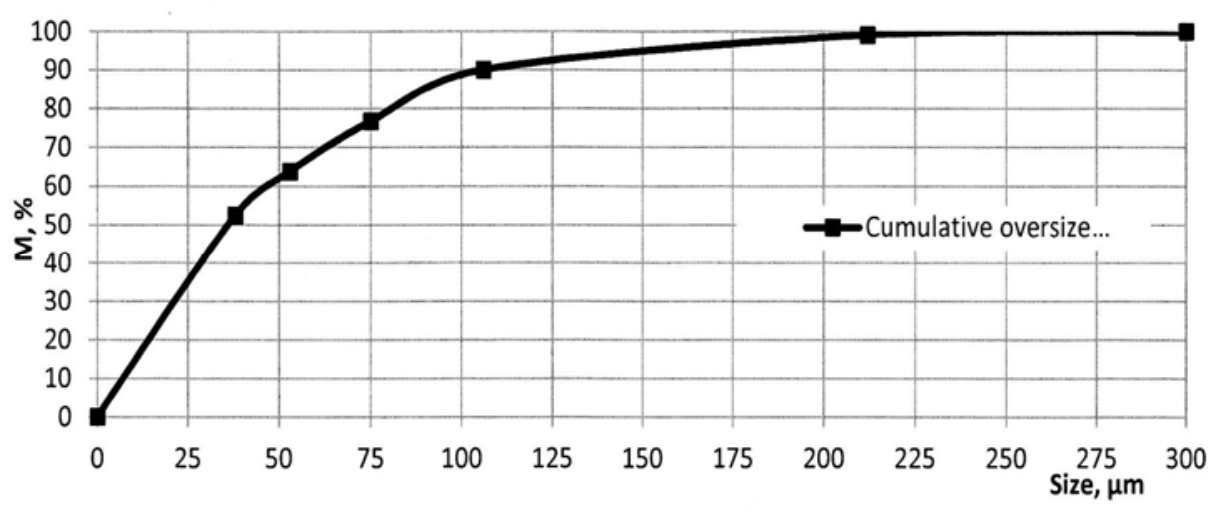

Figure 7 Grain-size distribution of grinded sample 


\section{Test Procedure}

Laboratory testing of lead minerals flotation concentration is carried out according to the following conditions:

- Pulp density in grinding processes was $70 \%$ of solid phase in all experiments,

- Grinding fineness was $75 \%-0.075$ $\mathrm{mm}$ (grain size that achieves an optimal degree of useful minerals realease, according to the mineralogical analysis),

- Pulp density in flotation processes was $30 \%$ of solid phase in all experiments,

- $\mathrm{pH}$ value of pulp in flotation process were 9 in all experiments,

- Potassium ethyl xanthate (PEX) and potassium amyl xanthate (PAX) were applied as collectors. Consumption of the collectors was $30 \mathrm{~g} / \mathrm{t}$ of both PEX and PAX in each experiment. The collector dose is determined based on the content of galena in the initial sample,

- Zinc sulphate $\left(\mathrm{ZnSO}_{4}\right)$ was applied as a depressant of sphalerite, with consumption of $60 \mathrm{~g} / \mathrm{t} \mathrm{ZnSO}_{4}$ per experiment,

- Sodium cyanide $(\mathrm{NaCN})$ was applied as a depressant of sulphide minerals of $\mathrm{As}, \mathrm{Fe}, \mathrm{Cu}$ and $\mathrm{Zn}$. Consumption of $\mathrm{NaCN}$ was $250 \mathrm{~g} / \mathrm{t}, 300 \mathrm{~g} / \mathrm{t}, 350 \mathrm{~g} / \mathrm{t}$ and $400 \mathrm{~g} / \mathrm{t}$ from the first to the fourth experiment, respectively,

- Dowfroth 250 was used as a frother in all experiments, with the dosage recommended by the manufacturer.

\section{RESULTS AND DISCUSSION}

Technological results of examination and material balances of the experiments are shown in Tables 2, 3, 4, and 5.

Table 2 Experiment 1: Technological results of examination

\begin{tabular}{|c|r|r|r|r|r|r|r|}
\hline Product & \multicolumn{1}{|c|}{$\mathbf{M}, \boldsymbol{\%}$} & \multicolumn{1}{|c|}{$\mathbf{P b}, \boldsymbol{\%}$} & $\mathbf{Z n}, \boldsymbol{\%}$ & \multicolumn{1}{|c|}{$\mathbf{A s}, \boldsymbol{\%}$} & $\mathbf{R}_{\mathbf{P b}}, \boldsymbol{\%}$ & $\mathbf{R}_{\mathbf{Z n}}, \boldsymbol{\%}$ & $\mathbf{R}_{\mathbf{A s}}, \boldsymbol{\%}$ \\
\hline $\mathbf{F}^{*}$ & 100.00 & 7.63 & 0.68 & 21.18 & 100.00 & 100.00 & 100.00 \\
\hline $\mathbf{R C}_{\mathbf{P b}}$ & 14.31 & 49.67 & 0.82 & 5.77 & 93.15 & 17.25 & 3.90 \\
\hline $\mathbf{T}$ & 85.69 & 0.61 & 0.66 & 23.75 & 6.85 & 82.75 & 96.10 \\
\hline
\end{tabular}

Table 3 Experiment 2: Technological results of examination

\begin{tabular}{|c|r|r|r|r|r|r|r|}
\hline Product & $\mathbf{M}, \boldsymbol{\%}$ & \multicolumn{1}{|c|}{$\mathbf{P b}, \boldsymbol{\%}$} & $\mathbf{Z n}, \boldsymbol{\%}$ & \multicolumn{1}{|c|}{$\mathbf{A s}, \boldsymbol{\%}$} & $\mathbf{R}_{\mathbf{P b}}, \boldsymbol{\%}$ & $\mathbf{R}_{\mathbf{Z n}}, \boldsymbol{\%}$ & $\mathbf{R}_{\mathbf{A s}}, \boldsymbol{\%}$ \\
\hline $\mathbf{F}$ & 100.00 & 7.14 & 0.69 & 20.46 & 100.00 & 100.00 & 100.00 \\
\hline $\mathbf{R C}_{\mathbf{P b}}$ & 15.17 & 43.04 & 1.12 & 4.08 & 91.44 & 24.62 & 3.02 \\
\hline $\mathbf{T}$ & 84.83 & 0.72 & 0.61 & 23.39 & 8.56 & 75.38 & 96.98 \\
\hline
\end{tabular}

Table 4 Experiment 3: Technological results of examination

\begin{tabular}{|c|r|r|r|r|r|r|r|}
\hline Product & $\mathbf{M}, \boldsymbol{\%}$ & \multicolumn{1}{|c|}{$\mathbf{P b}, \boldsymbol{\%}$} & \multicolumn{1}{|c|}{$\mathbf{Z n}, \boldsymbol{\%}$} & \multicolumn{1}{|c|}{$\mathbf{A s}, \boldsymbol{\%}$} & $\mathbf{R}_{\mathbf{P b}}, \boldsymbol{\%}$ & $\mathbf{R}_{\mathbf{Z n}}, \boldsymbol{\%}$ & $\mathbf{R}_{\text {As }}, \boldsymbol{\%}$ \\
\hline $\mathbf{F}$ & 100.00 & 7.35 & 0.65 & 20.62 & 100.00 & 100.00 & 100.00 \\
\hline $\mathbf{R C}_{\mathbf{P b}}$ & 13.86 & 48.74 & 1.07 & 3.93 & 91.91 & 22.82 & 2.64 \\
\hline $\mathbf{T}$ & 86.14 & 0.69 & 0.58 & 23.31 & 8.09 & 77.18 & 97.36 \\
\hline
\end{tabular}


Table 5 Experiment 4: Technological results of examination

\begin{tabular}{|c|r|r|r|r|r|r|r|}
\hline Product & $\mathbf{M}, \boldsymbol{\%}$ & \multicolumn{1}{|c|}{$\mathbf{P b}, \boldsymbol{\%}$} & \multicolumn{1}{|c|}{$\mathbf{Z n}, \boldsymbol{\%}$} & \multicolumn{1}{|c|}{$\mathbf{A s}, \boldsymbol{\%}$} & $\mathbf{R}_{\mathbf{P b}}, \boldsymbol{\%}$ & $\mathbf{R}_{\mathbf{Z n}}, \boldsymbol{\%}$ & $\mathbf{R}_{\text {As }}, \boldsymbol{\%}$ \\
\hline $\mathbf{F}$ & 100.00 & 7.09 & 0.71 & 20.83 & 100.00 & 100.00 & 100.00 \\
\hline $\mathbf{R C}_{\mathbf{P b}}$ & 14.27 & 45.48 & 0.98 & 2.19 & 91.54 & 19.69 & 1.50 \\
\hline $\mathbf{T}$ & 85.73 & 0.70 & 0.67 & 23.93 & 8.46 & 80.31 & 98.50 \\
\hline
\end{tabular}

$* F$-feed, $R C_{P b}-$ rough lead concentrate, $T$-tailings

Based on the results shown in the $\mathrm{Ta}-$ bles 2-5, it can be concluded that the highquality rough concentrate with a high lead recovery (over 90\%) can be obtained by described procedure. The enrichment ratio of lead ranges from 6.03 (experiment no. 2) to 6.63 (experiment no. 3 ).
Zinc content in the concentrate varies from $0.82 \%$ to $1.12 \%$. That is slightly higher than zinc content in the ore, but based on the recovery of zinc from tailings, conclusion is that the highest amount of sphalerite is successfully depressed during flotation process.

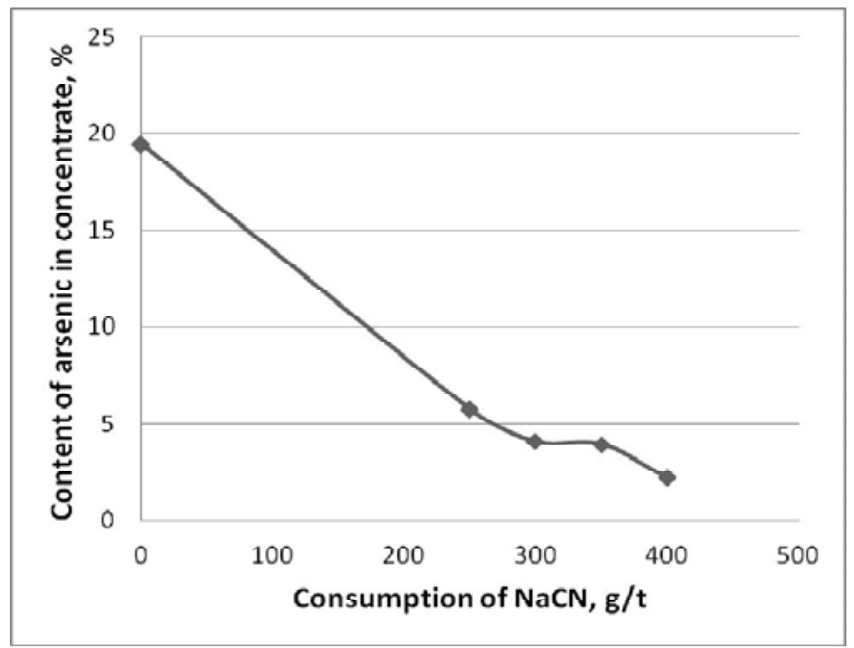

Figure 8 Arsenic content in concentrate in dependence on $\mathrm{NaCN}$ dosage

The amount of arsenic in a rough concentrate is significantly reduced, even up to 10 times (experiment no. 4), comparing to the amount of arsenic in the ore. Arsenic content in a rough concentrate is inversely proportional to the consumption of cyanide (Figure 8). That means the highest consumption of $\mathrm{NaCN}$ causes the smallest amount of arsenopyrite passing into the concentrate during flotation. Arsenic re covery from tailings is very high, ranging from $96.10 \%$ (experiment no. 1) to $98.50 \%$ (experiment no. 4). Based on the above, it follows that the arsenopyrite can be very successfully depressed using sodium cyanide as a depressant.

Finally, it should be noted that obtaining the high-quality commercial lead concentrate is possible only after the multiple cleaning of rough concentrate. 


\section{CONCLUSIONS}

The mineral arsenopyrite has distinguished flotation properties and in the presence of xanthates, within a wide range of pulp $\mathrm{pH}$ values (7-11), easily pass to concentrate (similarly to pyrite). Depression of arsenopyrite is not a simple procedure and does not always give satisfactory results. However, the results of these experimental tests showed that the depression of arsenopyrite can be successfully performed using sodium cyanide as a depressant. The required amount of $\mathrm{NaCN}$ during this procedure was $250-400 \mathrm{~g} / \mathrm{t}$, which is slightly higher than common dose of $\mathrm{NaCN}$ applied in the industrial flotation plants of lead-zinc ores.

\section{REFERENCES}

[1] S. Magdalinović, V. Marjanović, D. Urošević, D. Stanujkić, Flotation Concentration of Polymetallic Barite Ore, Mining Engineering 2(2012), ISSN 1451-0162, pp. 93-104;

[2] D. Milanović, S. Magdalinović, R. Jonović, Lj. Avramović, Choose Reagent for Obtain Low Content Concentrate of Scheelite, Mining Engineering 1(2009), ISSN 1451-0162, pp. 33-38;

[3] D. Draškić, (1975) Industrial Application of Mineral Processing, Publi- shing and Information Students Centre, Belgrade, p. 220 (in Serbian);

[4] D. Draškić, (1986) Industrial Application of Mineral Processing II book, Faculty of Mining and Geology, Belgrade, p. 300 (in Serbian);

[5] López Valdivieso, A., Sánchez López, A. A., Ojeda Escamilla, C., Fuerstenau, M. C. (2006) Flotation and Depression Control of Arsenopyrite through $\mathrm{pH}$ and Pulp Redox Potential using Xanthate as the Collector, International Journal of Mineral Processing, 81 (1), 27-34;

[6] Li, G. M., Zhang, H. E., Usui, S., (1992) Depression of Arsenopyrite in Alkaline Medium. International Journal of Mineral Processing, 34 (3), 253-257;

[7] Qian, X., Shinnosuke, U., Zhang, H. E. (1993) Depression of Arsenopyrite Flotation and its Separation from Pyrite in Guangxi Shanhu Tin Mine, XVIII International Mineral Processing Congress, pp. 23-28;

[8] Wang, X., Forssberg, E., Bolin, N. J. (1982). Activation of Arsenopyrites by Copper(II) Ions, Scandinavian Journal of Metallurgy, 18 (6), 288-294;

[9] Vreugde, M. J. A. (1982) Flotation Characteristics of Arsenopyrite, $\mathrm{PhD}$ Thesis, University of British Columbia. 


\begin{tabular}{ll}
\hline \hline INSTITUT ZA RUDARSTVO I METALURGIJU BOR & ISSN: 2334-8836 \\
& UDK: 622 \\
\hline \hline
\end{tabular}

Srđana Magdalinovic ${ }^{*}$, Ivana Jovanović, Vesna Ljubojev ${ }^{*}$

\section{UTICAJ NATRIJUM CIJANIDA NA SADRŽAJ ARSENA U FLOTACIJSKOM KONCENTRATU OLOVA**}

Izvod

Flotacijske osobine arsenopirita su slične flotacijskim osobinama pirita. Posebno u alkalnim uslovima (pH vrednost pulpe 7-11) i u prisustvu ksantata kao kolektora, arsenopirit lako prelazi u koncentrat. Dakle, ako ruda sadrži arsenopirit kao prateći mineral, njegove flotacijske osobine mogu izazvati poteškoće pri flotaciji drugih minerala (minerala $\mathrm{Pb}, \mathrm{Zn}, \mathrm{Sb}, \mathrm{W}$, itd.), jer se arsen smatra štetnim elementom $u$ većini koncentrata metaličnih minerala. U cilju dobijanja visoko kvalitetnih flotacijskih koncentrata, često je neophodno deprimirati arsenopirit što može biti uspešno ostvareno primenom cijanida.

Ovaj rad sadrži rezultate laboratorijskog ispitivanja izvedenog u cilju utvrđivanja mogućnosti dobijanja koncentrata olova sa niskim sadržajem arsena. Uzorak rude koji je korišćen u flotacijskim eksperimentima, sadržao je veliku količinu arsenopirita (preko $40 \%$ ). Rezultati pokazuju da sadržaj arsena u grubom koncentratu olova može biti umanjen $i$ do deset puta u odnosu na sadržaj u rudi korišćenjem $\mathrm{NaCN}$ kao deprimatora.

Ključne reči: arsen, natrijum cijanid, flotacijski koncentrat

\section{UVOD}

Arsen je štetan element u selektivnim koncentratima olova i cinka [1,2]. Dozvoljen sadržaj arsena u ovim koncentratima je predstavljen desetim delovima procenta [4].

Jedan od najznačajnijih minerala arsena je arsenopirit (FeAsS), koji je često glavni mineral jalovine u sulfidnim rudama. Prema tome, u pripremi polimetaličnih ruda olova, cinka, bakra, pirita i zlata, veoma je značajno da se ostvari uspešna deprimacija arsenopirita tokom izdvajanja u zasebne koncentrate. Draškić [3] naglašava da deprimaciju arsenopirita treba izvršiti na početku selektivne koncentracije jer je deprimaciju u kolektivnim koncentratima mnogo teže ostvariti.

Prema literaturnim podacima, deprimacija arsenopirita može da se ostvari u jako alkalnoj sredini $[6,7]$ i u prisustvu oksidanata, kao što je vodonik peroksid $\left(\mathrm{H}_{2} \mathrm{O}_{2}\right)$, kalijum permanganat $\left(\mathrm{KMnO}_{4}\right)$, mangan dioksid $\left(\mathrm{MnO}_{2}\right)$ i hipohlorna kiselina $(\mathrm{HClO})[3,5]$. Osim toga, neki autori navode da arsenopirit može uspešno da se deprimira natrijum cijanidom $(\mathrm{NaCN})[3,9]$. Šta više, mešavina amonijum-magnezijumovih soli može da bude uspešan deprimator. Uočeno je i da

\footnotetext{
* Institut za rudarstvo i metalurgiju Bor, srdjana.magdalinovic@irmbor.co.rs

** Ovaj rad je rezultat projekata: TR 33023: „,Razvoj tehnologija flotacijske koncentracije ruda bakra $i$ plemenitih metala radi postizanja boljih tehnoloških rezultata” i TR 34006: „Mehanohemijski tretman nedovoljno kvalitetnih mineralnih sirovina” finansiranim od strane Ministarstva za prosvetu, nauku i tehnološki razvoj Republike Srbije kojem ovim putem želimo da se zahvalimo.
} 
stroncijumovi i barijumovi joni mogu da unaprede deprimaciju arsenopirita i inhibiraju aktivaciju bakarnih jona. [5].

Arsenopirit flotira dobro uz upotrebu ksantata kao kolektora. Oksidacija jona ksantata $\left(\mathrm{X}^{-}\right)$do diksantogenata (X2) se opisuje kao mehanizam kroz koji se kolektor adsorbuje na međufazi arsenopirit/vodeni rastvor gde se površina arsenopirita ponaša kao katalizator za reakciju oksidacije obezbeđujući $\mathrm{OH}^{-}$jone kao proizvod reakcije [5]. Joni bakra (ukoliko su prisutni u flotacijskoj pulpi), aktiviraju flotaciju arsenopirita, snižavajući količinu ksantata potrebnog za flotaciju [3, 5, 8].
Cilj eksperimentalnih ispitivanja, čiji su rezultati prikazani u radu, bio je da se dobije grubi koncentrat olova sa niskim sadržajem arsena.

\section{EKSPERIMENTALNI DEO}

\section{Sirovina}

U eksperimentalnim ispitivanjima korišćen je uzorak polimetalične sulfidne rude. U cilju dobijanja odgovarajuće krupnoće, uzorak je prethodno izdrobljen u laboratorijskoj čeljusnoj drobilici, i zatim samleven u laboratorijskom mlinu sa kuglama. Hemijski sastav uzorka prikazan je u tablici 1.

Tabela 1. Hemijski sastav uzorka

\begin{tabular}{|c|c|c|c|c|c|c|c|c|}
\hline Komponenta & $\mathrm{Pb}$ & $\mathrm{Zn}$ & $\mathrm{Cu}$ & $\mathrm{As}$ & $\mathrm{Sb}$ & $\mathrm{S}$ & $\mathrm{Fe}$ & $\mathrm{SiO}_{2}$ \\
\hline Sadržaj, \% & 7,25 & 0,70 & 0,15 & 19,45 & 0,047 & 16,92 & 16,34 & 32,62 \\
\hline
\end{tabular}

$\mathrm{Na}$ osnovu rezultata prikazanih u tablici 1, može se zaključiti da ispitivani uzorak sadrži visoku količinu arsena, čak 19,45\%.

Kvalitativna mineraloška analiza uzorka izvedena je pomoću mikroskopa za propuštenu i odbijenu svetlost marke AXIOSKOP 40 sa jedinicom za mikrofotografiju. Mikroskopija je pokazala da uzorak sadrži sledeće glavne sulfidne minerale: arsenopirit $(43,53 \%)$, pirit $(34,71 \%)$, galenit $(5,88 \%)$ i sfalerit $(2,95 \%)$. Ostali sulfidi (halkopirit, halkozin) su prisutni u malim količinama.

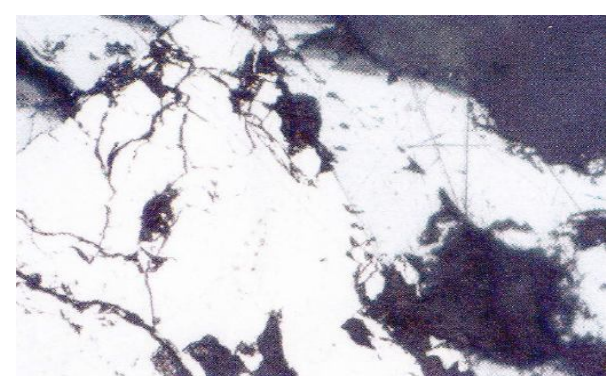

SI. 1. Sraslaci arsenopirita i galenita, uvećanje 200x, II N
Arsenopirit se uglavnom javlja u obliku krupnih kristalnih formi. U pojedinim zrnima arsenopirita zapažaju se sitna uklopljena zrna sfalerita. Takođe se zapažaju sraslaci arsenopirita sa piritom, galenitom i sfaleritom.

Galenit se javlja u vidu samostalnih krupnih zrna. Sitna zrna galenita vezana su sa piritom i arsenopiritom.

Slike 1-6 ilustruju rezultate mineraloške analize uzorka.

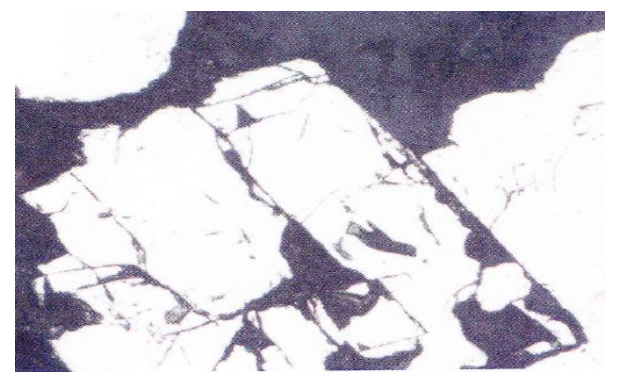

Sl. 2. Arsenopirit (rombične forme), uvećanje 200x, II N 


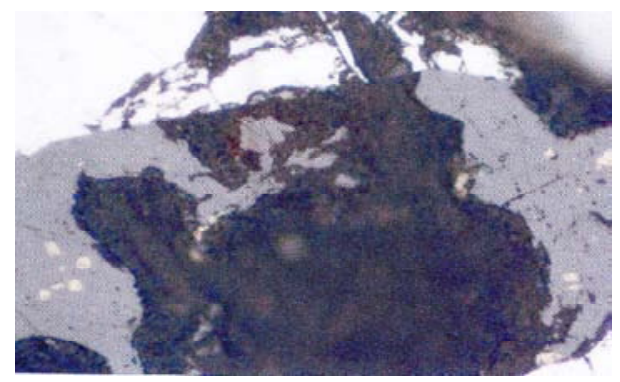

SI. 3. Izdvajanje halkopirita u sfaleritu, uvećanje 400x, II N

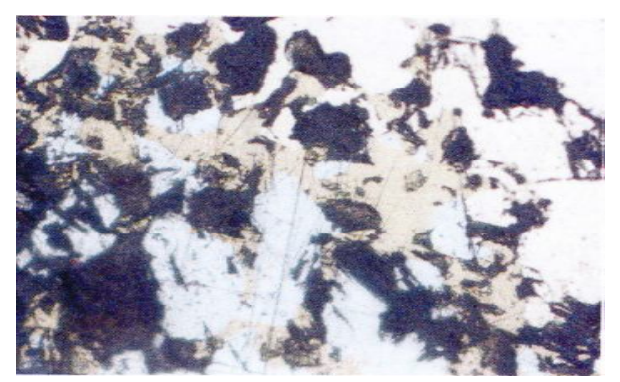

S1. 5. Sraslaci pirita, halkopirita i galenita, uvećanje 200x, II N

Za eksperimente flotacijske koncentracije bilo je neophodno samleti uzorak do odgovarajuće finoće $(75 \%-0,075 \mathrm{~mm})$.

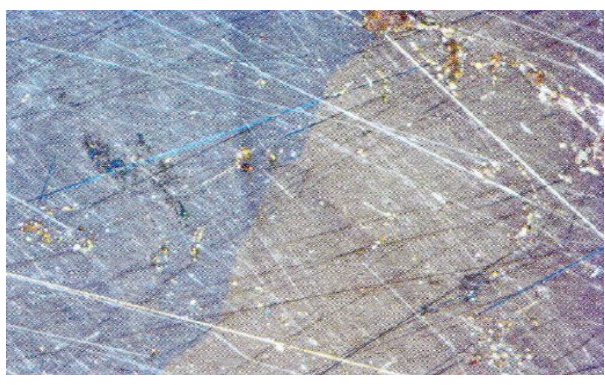

Sl. 4. Arsenopirit, uvećanje $100 x,+N$

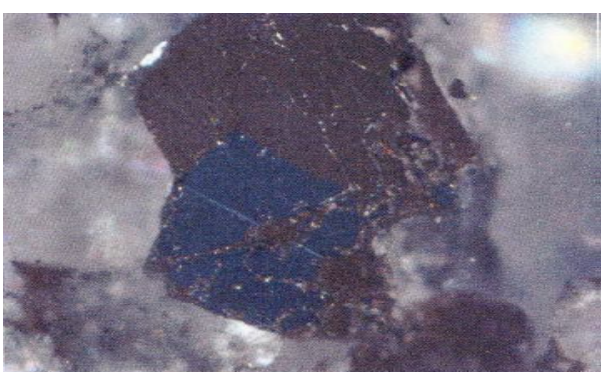

Sl. 6. Arsenopirit, uvećanje 200x, II N

Granulometrijski sastav samlevenog uzorka prikazan je na slici 7 .

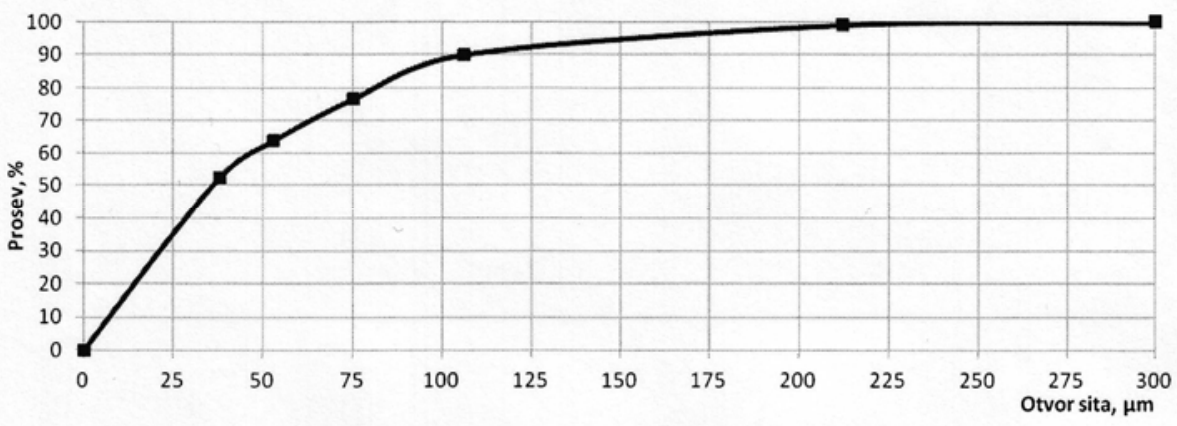

SI. 7. Granulometrijski sastav samlevenog uzorka

\section{Eksperimentalna procedura}

Laboratorijski eksperimenti flotacijske koncentracije minerala olova izvedeni su prema sledećim uslovima:
- Gustina pulpe u procesu mlevenja $70 \%$ čvrstog u svim eksperimentima 
- Finoća mlevenja 75\% - 0,075 mm (finoća mlevenja sa kojom je postignut optimalni stepen oslobađanja korisnih minerala prema mineraloškoj analizi)

- Gustina pulpe u procesu flotacijske koncentracije bila je 30\% čvrstog u svim eksperimentima

- pH vrednost pulpe u procesu flotacijske koncentracije bila je $9 \mathrm{u}$ svim eksperimentima

- korišćeni su kolektori kalijum etil ksantat (KEX) i kalijum amil ksantat (KAX) u dozi od po $30 \mathrm{~g} / \mathrm{t}$ u svim eksperimentima. Doza kolektora je utvrđena na osnovu sadržaja galenita u polaznom uzorku.

- Cinkl sulfat $\left(\mathrm{ZnSO}_{4}\right)$ je korišćen kao deprimator sfalerita $\mathrm{u}$ dozi od $60 \mathrm{~g} / \mathrm{t}$ $\mathrm{ZnSO}_{4}$ po eksperimentu.

- Natrijum cijanid $(\mathrm{NaCN})$ je primenjen kao deprimator sulfidnih minerala arsena, gvožđa, bakra i cinka. Potrošnja $\mathrm{NaCN}$ bila je $250 \mathrm{~g} / \mathrm{t}$, $300 \mathrm{~g} / \mathrm{t}, 350 \mathrm{~g} / \mathrm{t} \mathrm{i} 400 \mathrm{~g} / \mathrm{t}$ od prvog do četvrtog eksperimenta respektivno.

- Dowfroth 250 je korišćen kao penušač u svim eksperimentima u dozi koju preporučuje proizvođač.

Tabela 2. Eksperiment 1: Tehnološki rezultati ispitivanja i bilans koncentracije

\begin{tabular}{|c|c|c|c|c|c|c|c|}
\hline Proizvod & $\mathbf{m}, \boldsymbol{\%}$ & $\mathbf{P b}, \boldsymbol{\%}$ & $\mathbf{Z n}, \boldsymbol{\%}$ & $\mathbf{A s}, \boldsymbol{\%}$ & $\mathbf{I}_{\mathbf{P b}}, \boldsymbol{\%}$ & $\mathbf{I}_{\mathbf{Z n}}, \boldsymbol{\%}$ & $\mathbf{I}_{\mathbf{A s}}, \boldsymbol{\%}$ \\
\hline $\mathbf{U}^{*}$ & 100,00 & 7,63 & 0,68 & 21,18 & 100,00 & 100,00 & 100,00 \\
\hline $\mathbf{K}_{\mathbf{P b}}$ & 14,31 & 49,67 & 0,82 & 5,77 & 93,15 & 17,25 & 3,90 \\
\hline $\mathbf{O}$ & 85,69 & 0,61 & 0,66 & 23,75 & 6,85 & 82,75 & 96,10 \\
\hline
\end{tabular}

Tabela 3. Eksperiment 2: Tehnološki rezultati ispitivanja i bilans koncentracije

\begin{tabular}{|c|c|c|c|c|c|c|c|}
\hline Proizvod & $\mathbf{m}, \boldsymbol{\%}$ & $\mathbf{P b}, \boldsymbol{\%}$ & $\mathbf{Z n}, \boldsymbol{\%}$ & $\mathbf{A s}, \boldsymbol{\%}$ & $\mathbf{I}_{\mathbf{P b}}, \boldsymbol{\%}$ & $\mathbf{I}_{\mathbf{Z n}}, \boldsymbol{\%}$ & $\mathbf{I}_{\mathbf{A s}}, \boldsymbol{\%}$ \\
\hline $\mathbf{U}^{*}$ & 100,00 & 7,14 & 0,69 & 20,46 & 100,00 & 100,00 & 100,00 \\
\hline $\mathbf{K}_{\mathbf{P b}}$ & 15,17 & 43,04 & 1,12 & 4,08 & 91,44 & 24,62 & 3,02 \\
\hline $\mathbf{O}$ & 84,83 & 0,72 & 0,61 & 23,39 & 8,56 & 75,38 & 96,98 \\
\hline
\end{tabular}

Tabela 4. Eksperiment 3: Tehnološki rezultati ispitivanja i bilans koncentracije

\begin{tabular}{|c|c|c|c|c|c|c|c|}
\hline Proizvod & $\mathbf{m}, \boldsymbol{\%}$ & $\mathbf{P b}, \boldsymbol{\%}$ & $\mathbf{Z n , \%}$ & $\mathbf{A s}, \boldsymbol{\%}$ & $\mathbf{I}_{\mathbf{P b}}, \boldsymbol{\%}$ & $\mathbf{I}_{\mathbf{Z n}}, \boldsymbol{\%}$ & $\mathbf{I}_{\mathbf{A s}}, \boldsymbol{\%}$ \\
\hline $\mathbf{U}^{*}$ & 100,00 & 7,35 & 0,65 & 20,62 & 100,00 & 100,00 & 100,00 \\
\hline $\mathbf{K}_{\mathbf{P b}}$ & 13,86 & 48,74 & 1,07 & 3,93 & 91,91 & 22,82 & 2,64 \\
\hline $\mathbf{O}$ & 86,14 & 0,69 & 0,58 & 23,31 & 8,09 & 77,18 & 97,36 \\
\hline
\end{tabular}

Tabela 5. Eksperiment 4: Tehnološki rezultati ispitivanja i bilans koncentracije

\begin{tabular}{|c|c|c|c|c|c|c|c|}
\hline Proizvod & $\mathbf{m}, \boldsymbol{\%}$ & $\mathbf{P b}, \boldsymbol{\%}$ & $\mathbf{Z n}, \boldsymbol{\%}$ & $\mathbf{A s}, \boldsymbol{\%}$ & $\mathbf{I}_{\mathbf{P b}}, \boldsymbol{\%}$ & $\mathbf{I}_{\mathbf{Z n}}, \boldsymbol{\%}$ & $\mathbf{I}_{\mathbf{A s}}, \boldsymbol{\%}$ \\
\hline $\mathbf{U}^{*}$ & 100,00 & 7,09 & 0,71 & 20,83 & 100,00 & 100,00 & 100,00 \\
\hline $\mathbf{K}_{\mathbf{P b}}$ & 14,27 & 45,48 & 0,98 & 2,19 & 91,54 & 19,69 & 1,50 \\
\hline $\mathbf{O}$ & 85,73 & 0,70 & 0,67 & 23,93 & 8,46 & 80,31 & 98,50 \\
\hline
\end{tabular}

*U - Polazni uzorak, $\mathbf{K}_{\mathbf{P b}}$ - grubi koncentrat olova, $O$ - otok flotacije olova 
$\mathrm{Na}$ osnovu rezultata prikazanih $\mathrm{u}$ tablicama 2-5, može se zaključiti da visoko kvalitetni grubi koncentrat olova sa visokim iskorišćenjem olova može da se dobije pomoću opisane procedure. Stepen obogaćivanja olova ide i do 6,03 (eksperiment 2) i 6,63 (eksperiment 3).
Sadržaj cinka u koncentratu varira od $0,82 \%$ do $1,12 \%$. To je nešto više od sadržaja cinka u polaznom uzorku, ali u odnosu na raspodelu cinka u otoku flotacije olova, zaključak je da je veći deo sfalerita uspešno deprimiran tokom flotacijskog procesa.

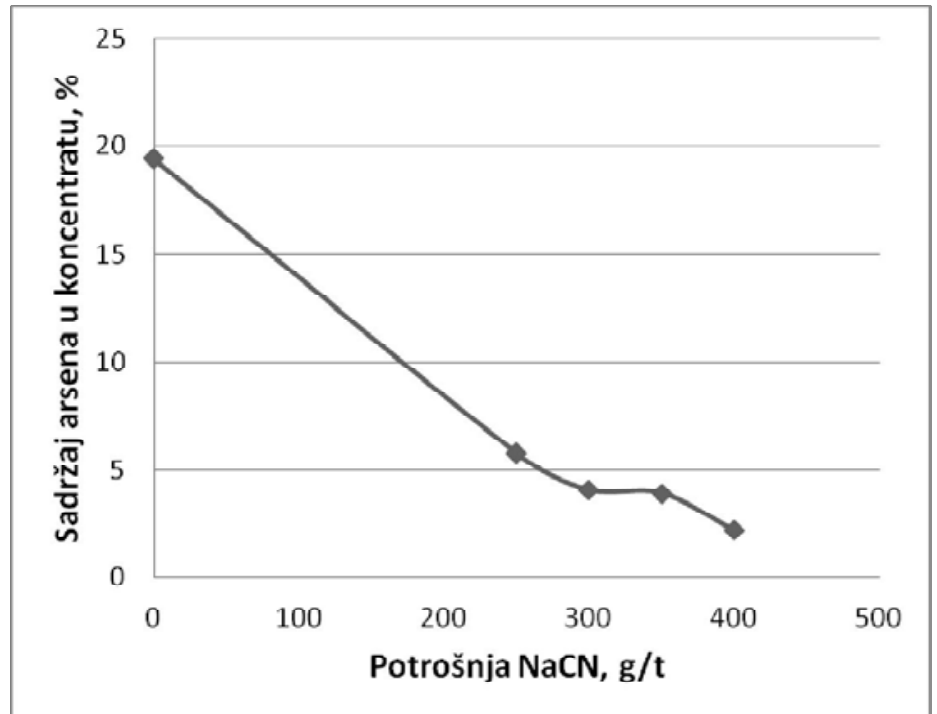

Sl. 8. Sadržaj arsena u koncentratu u zavisnosti od potrošnje NaCN

Sadržaj arsena u grubom koncentratu olova značajno je redukovan čak i do deset puta (eksperiment 4), upoređujući sa sadržajem arsena u rudi. Sadržaj arsena u grubom koncentratu olova je obrnuto proporcionalan potrošnji cijanida. To znači da najveća potrošnja $\mathrm{NaCN}$ uzrokuje da najmanja količina arsenopirita tokom flotacijske koncentracije pređe $\mathrm{u}$ koncentrat. Raspodela arsena u otoku flotacije olova je veoma visoka i kreće se od 96,10\% (eksperiment 1) do $98,50 \%$ (eksperiment 4). $\mathrm{Na}$ osnovu prethodnog, vidi se da arsenopirit može biti veoma uspešno deprimiran upotrebom natrijum cijanida kao deprimatora.

$\mathrm{Na}$ kraju treba napomenuti da je dobijanje visokokvalitetnog tržišnog koncentrata olova moguće samo posle prečišćavanja grubog koncentrata olova.

\section{ZAKLJUČAK}

Mineral arsenopirit ima izražene flotacijske osobine i u prisustvu ksantata, unutar širokog opsega $\mathrm{pH}$ vrednosti pulpe (7-11), lako prelazi u koncentrat (slično piritu). Deprimacija arsenopirita nije jednostavna metoda i ne daje uvek zadovoljavajuće rezultate. Međutim, rezultati eksperimenata pokazuju da deprimacija arsenopirita može biti uspešno izvedena upotrebom natrijum cijanida kao deprimatora. Potrebna količina $\mathrm{NaCN}$ za opisanu procedure bila je 250-400 g/t, što je 
nešto više od uobičajene doze $\mathrm{NaCN}$ koja se primenjuje $\mathrm{u}$ industrijskim flotacijskim postrojenjima za olovo cinkove rude.

\section{LITERATURA}

[1] S. Magdalinović, V. Marjanović, D. Urošević, D. Stanujkić, Flotacijska koncentracija polimetalične baritske rude, Rudarski radovi 2/2012, ISSN 1451-0162, str. 93-104.

[2] D. Milanović, S. Magdalinović, R. Jonović, Lj. Avramović, Izbor reagensa za dobijanje niskosadržajnog koncentrata šelita, Rudarski radovi 1/2009, ISSN 1451-0162, str. 33-38.

[3] D. Draškić, Industrijska primena pripreme mineralnih sirovina, Izdavačko informativni centar studenata, Beograd, 1975, str. 220.

[4] D. Draškić, Industrijska primena pripreme mineralnih sirovina, Rudarsko geološki fakultet, Beograd, 1986, str. 300 .
[5] López Valdivieso, A., Sánchez López, A. A., Ojeda Escamilla, C., Fuerstenau, M. C., Flotation and depression control of arsenopyrite through $\mathrm{pH}$ and pulp redox potential using xanthate as the collector. International Journal of Mineral Processing, 81 (1), 2006, pp. 27-34.

[6] Li, G.M., Zhang, H.E., Usui, S., Depression of arsenopyrite in alkaline medium. International Journal of Mineral Processing, 34 (3), 1992, pp. 253-257.

[7] Qian, X., Shinnosuke, U., Zhang, H. E., Depression of arsenopyrite flotation and its separation from pyrite in Guangxi Shanhu Tin Mine. XVIII International Mineral Processing Congress, 1993, pp. 23-28.

[8] Wang, X., Forssberg, E., Bolin, N.J., Activation of arsenopyrites by copper(II) ions. Scandinavian Journal of Metallurgy, 18 (6), 1982, pp. 288-294.

[9] Vreugde, M. J. A., Flotation characteristics of arsenopyrite, $\mathrm{PhD}$ Thesis, University of British Columbia, 1982 